\title{
Novel fat-link fermion actions
}

J. M. Zanotti ${ }^{\text {, }}$ S. Bilson-Thompson ${ }^{\mathrm{a}}$, F. D. R. Bonnet ${ }^{\mathrm{a}}$, P. D. Coddington ${ }^{\mathrm{a}}$, D. B. Leinweber ${ }^{\mathrm{a}}$, A. G. Williams ${ }^{\text {a }}$ J. B. Zhang ${ }^{\mathrm{a}}$, W. Melnitchouk ${ }^{\mathrm{a}, \mathrm{b}}$ and F. X. Lee $\mathrm{L}^{\mathrm{b}, \mathrm{c}}$

aSpecial Research Centre for the Subatomic Structure of Matter, and

Department of Physics and Mathematical Physics, Adelaide University, Adelaide 5005, Australia

${ }^{\mathrm{b}}$ Jefferson Lab, 12000 Jefferson Avenue, Newport News, VA 23606, U.S.A.

${ }^{c}$ Center for Nuclear Studies, Department of Physics,

The George Washington University, Washington, D.C. 20052, U.S.A.

The hadron mass spectrum is calculated in lattice QCD using a novel fat-link clover fermion action in which only the irrelevant operators of the fermion action are constructed using smeared links. The simulations are performed on a $16^{3} \times 32$ lattice with a lattice spacing of $a=0.125 \mathrm{fm}$. We compare actions with $n=4$ and 12 smearing sweeps with a smearing fraction of 0.7 . The $n=4$ Fat-Link Irrelevant Clover (FLIC) action provides scaling which is superior to mean-field improvement, and offers advantages over nonperturbative $\mathcal{O}(a)$ improvement.

\section{INTRODUCTION}

Understanding the generation of hadron mass from first principles has proved to be challenging. The only method for deriving hadron masses directly from QCD is a numerical calculation on the lattice. The high computational cost required to perform accurate lattice calculations at small lattice spacings, however, has led to an increased interest in quark action improvement. To avoid the famous doubling problem, Wilson fermions [1] introduce additional terms which explicitly break chiral symmetry at $\mathcal{O}(a)$. To extrapolate reliably to the continuum, simulations must be performed on fine lattices, which are computationally very expensive. The scaling properties of the Wilson action at finite $a$ can be improved by introducing any number of irrelevant operators of increasing dimension which vanish in the continuum limit.

The Sheikholeslami-Wohlert (clover) action [2] introduces an additional irrelevant dimension-five operator to the standard Wilson [1] quark action,

$S_{\mathrm{SW}}=S_{\mathrm{W}}-\frac{i a C_{\mathrm{SW}} r}{4} \bar{\psi}(x) \sigma_{\mu \nu} F_{\mu \nu} \psi(x)$

where $S_{\mathrm{W}}$ is the standard Wilson action and $C_{\mathrm{SW}}$ is the clover coefficient which can be tuned to remove $\mathcal{O}(a)$ artifacts. Nonperturbative (NP) $\mathcal{O}(a)$ improvement [3] tunes $C_{\mathrm{SW}}$ to all powers in $g^{2}$ and displays excellent scaling, as shown by Edwards et al. [4] who studied the scaling properties of the nucleon and vector meson masses for various lattice spacings (see also Section 4 below). In particular, the linear behavior of the NPimproved clover actions, when plotted against $a^{2}$, demonstrates that $\mathcal{O}(a)$ errors are removed. It was also found in Ref. [4] that a linear extrapolation of the mean-field improved data fails, indicating that $\mathcal{O}(a)$ errors are still present.

A drawback to the clover action, however, is the associated problem of exceptional configurations, where the quark propagator encounters singular behavior as the quark mass becomes small. In practice, this prevents the use of coarse lattices $(\beta<5.7 \sim a>0.18 \mathrm{fm})[5,6]$. Furthermore, the plaquette version of $F_{\mu \nu}$, which is commonly used in Eq. (1), has large $\mathcal{O}\left(a^{2}\right)$ errors, which can lead to errors of the order of $10 \%$ in the topological charge even on very smooth configurations [7].

The idea of using fat links in fermion actions was first explored by the MIT group [8] and more recently has been studied by DeGrand et al. $[6,9]$, who showed that the exceptional configuration problem can be overcome by using a fat-link (FL) clover action. Moreover, the renormalization of 
the coefficients of action improvement terms are small. A drawback to conventional fat-link techniques, however, is that in smearing the links gluon interactions are removed at the scale of the cutoff. While this has some tremendous benefits, the short-distance quark interactions are lost. As a result decay constants, which are sensitive to the wave function at the origin, are suppressed.

A solution to these problems is to work with two sets of links in the fermion action. In the relevant dimension-four operators, one works with the untouched links generated via Monte Carlo methods, while the smeared fat links are introduced only in the higher dimension irrelevant operators. In this way the continuum limit of the theory is perfectly well defined.

In this talk I will present the first results of simulations of the spectrum of light mesons and baryons using this variation on the clover action. In particular, we will start with the standard clover action and replace the links in the irrelevant operators with APE smeared [10], or fat links. We shall refer to this action as the FatLink Irrelevant Clover (FLIC) action. Although the idea of using fat links only in the irrelevant operators of the fermion action was developed here independently, suggestions have appeared previously [11]. To the best of our knowledge, this is the first report of lattice QCD calculations using this novel fermion action.

\section{GAUGE ACTION}

The simulations are performed using a treelevel $\mathcal{O}\left(a^{2}\right)$-Symanzik-improved [12] gauge action on a $16^{3} \times 32$ lattice at $\beta=4.60$, providing a lattice spacing $a=0.125(2) \mathrm{fm}$ determined from the string tension with $\sqrt{\sigma}=440 \mathrm{MeV}$. A total of 50 configurations are used in this analysis, and the error analysis is performed by a third-order, single-elimination jackknife, with the $\chi^{2}$ per degree of freedom $\left(N_{D F}\right)$ obtained via covariance matrix fits.

\section{FAT-LINK IRRELEVANT FERMION ACTION}

Fat links $[6,9]$ are created by averaging or smearing links on the lattice with their near- est neighbors in a gauge covariant manner (APE smearing). The smearing procedure [10] replaces a link, $U_{\mu}(x)$, with a sum of the link and $\alpha$ times its staples

$$
\begin{gathered}
U_{\mu}(x) \quad \rightarrow \quad U_{\mu}^{\prime}(x)=(1-\alpha) U_{\mu}(x) \\
+\frac{\alpha}{6} \sum_{\substack{\nu=1 \\
\nu \neq \mu}}^{4}\left[U_{\nu}(x) U_{\mu}(x+\nu a) U_{\nu}^{\dagger}(x+\mu a)\right. \\
\left.+U_{\nu}^{\dagger}(x-\nu a) U_{\mu}(x-\nu a) U_{\nu}(x-\nu a+\mu a)\right],
\end{gathered}
$$

followed by projection back to $\mathrm{SU}(3)$. We select the unitary matrix $U_{\mu}^{\mathrm{FL}}$ which maximizes

$$
\mathcal{R} e \operatorname{tr}\left(U_{\mu}^{\mathrm{FL}} U_{\mu}^{\prime \dagger}\right)
$$

by iterating over the three diagonal $\mathrm{SU}(2)$ subgroups of $\mathrm{SU}(3)$. We repeat this procedure of smearing followed immediately by projection $n$ times. We create our fat links by setting $\alpha=0.7$ and comparing $n=4$ and 12 smearing sweeps. The mean-field improved FLIC action now becomes

$S_{\mathrm{SW}}^{\mathrm{FL}}=S_{\mathrm{W}}^{\mathrm{FL}}-\frac{i C_{\mathrm{SW}} \kappa r}{2\left(u_{0}^{\mathrm{FL}}\right)^{4}} \bar{\psi}(x) \sigma_{\mu \nu} F_{\mu \nu} \psi(x)$,

where $F_{\mu \nu}$ is constructed using fat links, and where the mean-field improved Fat-Link Irrelevant Wilson action is

$$
\begin{aligned}
S_{\mathrm{W}}^{\mathrm{FL}} & =\sum_{x} \bar{\psi}(x) \psi(x) \\
+ & \kappa \sum_{x, \mu} \bar{\psi}(x)\left[\gamma _ { \mu } \left(\frac{U_{\mu}(x)}{u_{0}} \psi(x+\hat{\mu})\right.\right. \\
& \left.-\frac{U_{\mu}^{\dagger}(x-\hat{\mu})}{u_{0}} \psi(x-\hat{\mu})\right) \\
- & r\left(\frac{U_{\mu}^{\mathrm{FL}}(x)}{u_{0}^{\mathrm{FL}}} \psi(x+\hat{\mu})\right. \\
& \left.\left.+\frac{U_{\mu}^{\mathrm{FL} \dagger}(x-\hat{\mu})}{u_{0}^{\mathrm{FL}}} \psi(x-\hat{\mu})\right)\right],
\end{aligned}
$$

with $\kappa=1 /(2 m+8 r)$. We take the standard value $r=1$. The $\gamma$-matrices are hermitian and $\sigma_{\mu \nu}=\left[\gamma_{\mu}, \gamma_{\nu}\right] /(2 i)$.

As reported in Table 1, the mean-field improvement parameter for the fat links is very close to 1. Hence, the mean-field improved coefficient for 
Table 1

The value of the mean link for different numbers of smearing sweeps, $n$.

\begin{tabular}{ccc}
\hline$n$ & $u_{0}^{\mathrm{FL}}$ & $\left(u_{0}^{\mathrm{FL}}\right)^{4}$ \\
\hline 0 & 0.88894473 & 0.62445197 \\
4 & 0.99658530 & 0.98641100 \\
12 & 0.99927343 & 0.99709689 \\
\hline
\end{tabular}

$C_{\mathrm{SW}}$ is expected to be adequate ${ }^{1}$. In addition, actions with many irrelevant operators (e.g. the $\mathrm{D}_{234}$ action) can now be handled with confidence as tree-level knowledge of the improvement coefficients should be sufficient. Another advantage is that one can now use highly improved definitions of $F_{\mu \nu}$ (involving terms up to $u_{0}^{12}$ ), which give impressive near-integer results for the topological charge [13].

In particular, we employ an $\mathcal{O}\left(a^{4}\right)$ improved definition of $F_{\mu \nu}$ [13] in which the standard cloversum of four $1 \times 1$ Wilson loops lying in the $\mu, \nu$ plane is combined with $2 \times 2$ and $3 \times 3$ Wilson loop clovers.

Work by DeForcrand et al. [14] suggests that 7 cooling sweeps are required to approach topological charge within $1 \%$ of integer value. This is approximately $16 \mathrm{APE}$ smearing sweeps at $\alpha=0.7[15]$. However, achieving integer topological charge is not necessary for the purposes of studying hadron masses, as has been well established. To reach integer topological charge, even with improved definitions of the topological charge operator, requires significant smoothing and associated loss of short-distance information. Instead, we regard this as an upper limit on the number of smearing sweeps.

Using unimproved gauge fields and an unimproved topological charge operator, Bonnet et al. [7] found that the topological charge settles down after about 10 sweeps of APE smearing at $\alpha=0.7$. Consequently, we create fat links with APE smearing parameters $n=12$ and $\alpha=0.7$.

\footnotetext{
${ }^{1}$ Our experience with topological charge operators suggests that it is advantageous to include $u_{0}$ factors, even as they approach 1.
}

This corresponds to $\sim 2.5$ times the smearing used in Refs. $[6,9]$. Further investigation reveals that improved gauge fields with a small lattice spacing $(a=0.125 \mathrm{fm})$ are smooth after only 4 sweeps. Hence, we perform calculations with 4 sweeps of smearing at $\alpha=0.7$ and consider $n=12$ as a second reference. Table 1 lists the values of $u_{0}^{\mathrm{FL}}$ for $n=0,4$ and 12 smearing sweeps.

A fixed boundary condition is used for the fermions by setting

$U_{t}(\vec{x}, n t)=0$ and $U_{t}^{\mathrm{FL}}(\vec{x}, n t)=0 \quad \forall \vec{x}$

in the hopping terms of the fermion action. The fermion source is centered at the space-time location $(x, y, z, t)=(1,1,1,3)$, which allows for two steps backward in time without loss of signal. Gauge-invariant gaussian smearing [16] in the spatial dimensions is applied at the source to increase the overlap of the interpolating operators with the ground states.

\section{RESULTS}

Hadron masses are extracted from the Euclidean time dependence of the calculated two point correlation functions. The effective masses are given by

$M(t+1 / 2)=\log [G(t)]-\log [G(t+1)]$.

The critical value of $\kappa, \kappa_{c}$, is determined by linearly extrapolating $m_{\pi}^{2}$ as a function of $m_{q}$ to zero. We used five values of quark mass and the strange quark mass was taken to be the second heaviest quark mass.

Effective masses (6) are calculated as a function of time and various time-fitting intervals are tested with a covariance matrix to obtain $\chi^{2} / N_{\mathrm{DF}}$. Good values of $\chi^{2} / N_{\mathrm{DF}}$ are obtained for many different time-fitting intervals as long as one fits after time slice 8 . All fits for this action are therefore performed on time slices 9 through 14. For the Wilson action and the FLIC action with $n=12$ ("FLIC12") the fitting regimes used are $9-13$ and $9-14$, respectively.

The behavior of the $\rho$, nucleon and $\Delta$ masses as a function of squared pion mass is shown in Fig. 1 for the various actions. The first feature to note is the excellent agreement between the FLIC4 and 


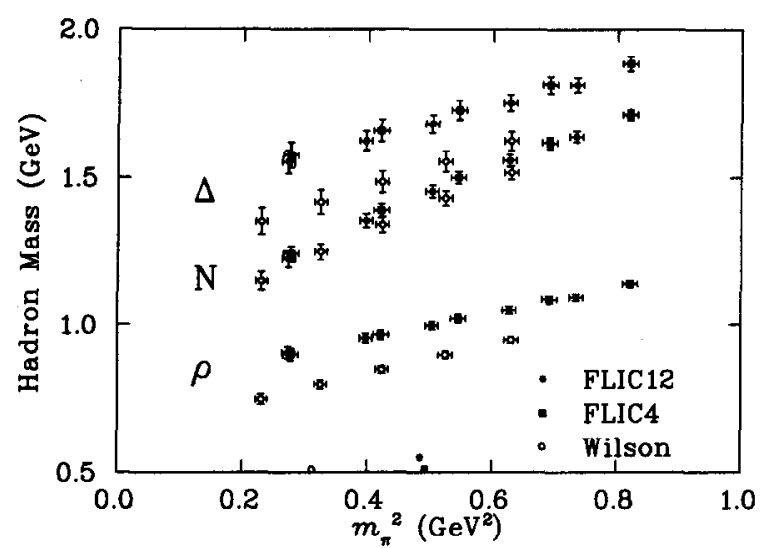

Figure 1. Masses of the nucleon, $\Delta$ and $\rho$ meson versus $m_{\pi}^{2}$ for the FLIC4, FLIC12 and Wilson actions.

FLIC12 actions. On the other hand, the Wilson action appears to lie somewhat low in comparison. It is also reassuring that all actions give the correct mass ordering in the spectrum. The value of the squared pion mass at $m_{\pi} / m_{\rho}=0.7$ is plotted on the abscissa for the three actions as a reference point. This point is chosen in order to allow comparison of different results by interpolating them to a common value of $m_{\pi} / m_{\rho}=0.7$, rather than extrapolating them to smaller quark masses, which is subject to larger systematic and statistical uncertainties.

The scaling behavior of the different actions is illustrated in Fig. 2. The present results for the Wilson action agree with those of Ref. [4]. The first feature to observe in Fig. 2 is that actions with fat-link irrelevant operators perform extremely well. For both the vector meson and the nucleon, the FLIC4 action performs systematically better than the FLIC12. This suggests that 12 smearing sweeps removes too much shortdistance information from the gauge-field configurations. On the other hand, 4 sweeps of smearing combined with our $\mathcal{O}\left(a^{4}\right)$ improved $F_{\mu \nu}$ provides excellent results, without the fine tuning of $C_{\mathrm{SW}}$ in the NP improvement program. Notice that for the $\rho$ meson, a linear extrapolation of the mean-

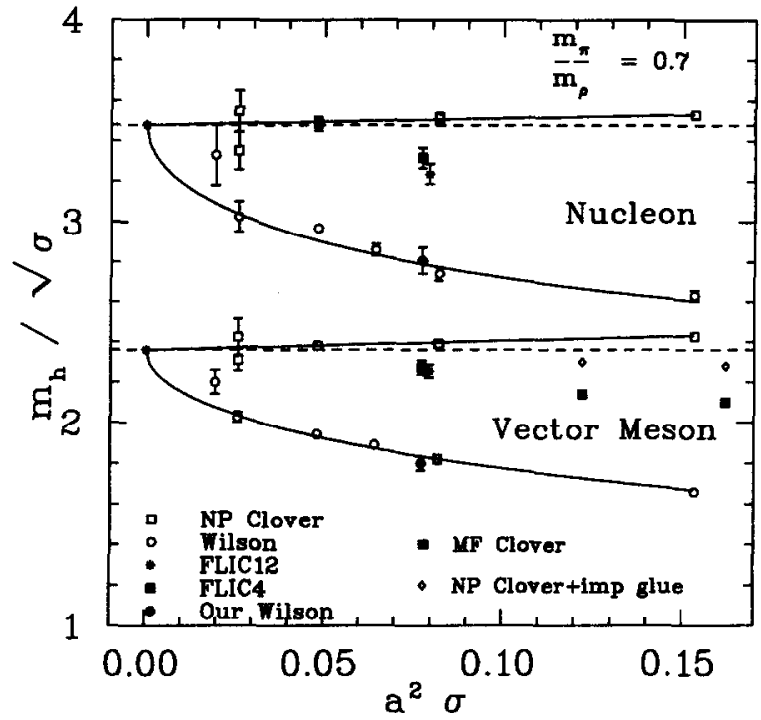

Figure 2. Nucleon and vector meson masses for the Wilson, NP-improved and FLIC actions obtained by interpolating our results of Fig. 1 to $m_{\pi} / m_{\rho}=0.7$. Results from the present simulations are indicated by the solid points. The fat links are constructed with $n=4$ (solid squares) and $n=12$ (stars) smearing sweeps at $\alpha=0.7$.

field improved clover points in Fig. 2 indicates that there is better improvement when using fat links in the irrelevant operators. While there are no NP-improved clover plus improved glue simulation results at $a^{2} \sigma \sim 0.08$, the simulation results that are available indicate that the fat-link results also compete well with those obtained with a NPimproved clover fermion action.

Finally, we compare the convergence rates of the different actions by comparing the number of Stabilised Biconjugate Gradient (BiCGStab) [17] iterations required to invert the fermion matrix. The FLIC12 action converges at a slightly faster rate to the Wilson fermion action and the FLIC4 action is the clear winner due to the small number of BiCGStab iterations required to invert the fermion matrix. This provides great promise for performing simulations at quark masses closer to 
the physical values.

\section{CONCLUSIONS}

We have examined the hadron mass spectrum using a novel Fat-Link Irrelevant Clover (FLIC) fermion action, in which only the irrelevant, higher-dimension operators involve smeared links. One of the main conclusions of this work is that the use of fat links in the irrelevant operators provides excellent results. Fat links promise improved scaling behavior over mean-field improvement. This technique also solves a significant problem with $\mathcal{O}(a)$ nonperturbative improvement on mean field-improved gluon configurations. Simulations are possible and the results are competitive with nonperturbative-improved clover results on plaquette-action gluon configurations. We have found that minimal smearing holds the promise of better scaling behavior. Our results suggest that too much smearing removes relevant information from the gauge fields, leading to poorer performance. Fermion matrix inversion for FLIC4 is more efficient and results show no sign of exceptional configuration problems.

This work paves the way for promising future studies. It will be of great interest to consider different lattice spacings to further test the scaling of the fat-link actions. Furthermore, the exceptional configuration issue can be explored by pushing the quark mass down to lower values. The $n=\mathbf{4}$ FLIC action holds great promise for circumventing this issue as evidenced by the relative ease with which one can invert the fermion matrix. A study of the spectrum of excited hadrons using the fat-link clover actions is currently in progress [18].

This work was supported by the Australian Research Council. We would also like to thank the National Computing Facility for Lattice Gauge Theories for the use of the Orion Supercomputer. W.M. and F.X.L. were partially supported by the U.S. Department of Energy contract DE-AC05-84ER40150, under which the Southeastern Universities Research Association (SURA) operates the Thomas Jefferson National Accelerator Facility (Jefferson Lab).

\section{REFERENCES}

1. K. G. Wilson, in New Phenomena in Subnuclear Physics, Part A, A. Zichichi (ed.), Plenum Press, New York, p. 69, 1975.

2. B. Sheikholeslami and R. Wohlert, Nucl. Phys. B259 (1985) 572.

3. M. Luscher et al., Nucl. Phys. B478 (1996) 365 [hep-lat/9605038]; M. Luscher et al., Nucl. Phys. B491 (1997) 323, 344 [heplat/9609035].

4. R.G.Edwards, U.M.Heller and T.R.Klassen, Phys. Rev. Lett. 80 (1998) 3448 [heplat/9711052]; see also R. D. Kenway, Nucl. Phys. Proc. Suppl. 73 (1999) 16 [heplat $/ 9810054$ ] for a review.

5. W. Bardeen et al., Phys. Rev. D 57 (1998) 1633 [hep-lat/9705008]; W. Bardeen et al., Phys. Rev. D 57 (1998) 3890.

6. T. DeGrand et al. (MILC Collaboration) [hep-lat/9807002].

7. F. D. Bonnet et al., Phys. Rev. D 62 (2000) 094509 [hep-lat/0001018].

8. M. C. Chu et al., Phys. Rev. D 49 (1994) 6039 [hep-lat/9312071].

9. T. DeGrand (MILC collaboration), Phys. Rev. D 60 (1999) 094501 [hep-lat/9903006].

10. M. Falcioni et al., Nucl. Phys. B251 (1985) 624; M. Albanese et al., Phys. Lett. B 192 (1987) 163.

11. H. Neuberger, Phys. Rev. D 61, 085015 (2000) [arXiv:hep-lat/9911004].

12. K. Symanzik, Nucl. Phys. B226 (1983) 187.

13. S. Bilson-Thompson et al., these proceedings; and in preparation.

14. P. de Forcrand et al., Nucl. Phys. B499 (1997) 409 [hep-lat/9701012]; P. de Forcrand et al., [hep-lat/9802017].

15. F. D. Bonnet et al., [hep-lat/0106023].

16. S. Gusken, Nucl. Phys. Proc. Suppl. 17, 361 (1990).

17. A. Frommer et al., Int. J. Mod. Phys. C 5 (1994) 1073 [hep-lat/9404013]

18. W. Melnitchouk et al., these proceedings; and in preparation. 